\title{
New eating disorder service
}

\author{
Harry R. Millar
}

\begin{abstract}
Aims and method The paper describes the first three years experience of a new out-patient eating disorder service for adults. Clinical data on referrals using standardised assessments are presented.

Results The referral rate rapidly rose to over 200 patients per annum. Forty-nine per cent of referrals were bulimic disorders and $26 \%$ had either anorexia nervosa or atypical anorexia nervosa. Seventy per cent were directly referred by general practitioners.

Clinical implications The creation of a specialist eating disorder senvice quickly leads to a high referral rate suggesting a previously unmet need. A service based on assessment and treatment by nurse therapists is acceptable and feasible with appropriate training and support from psychiatry, clinical psychology and dietetics. Expectations of a specialist service include teaching. consultation, research and audit as well as the provision of direct clinical care.
\end{abstract}

A Royal College of Psychiatrists (1992) report recommends that across the country "a consultant-led local service for eating disorders should be established, together with appropriate non-medial support." In 1994, an adult outpatient eating disorder service of this nature was funded for the Grampian Health Board Area (population 533 000). We think our early experience will help others setting up similar services elsewhere.

The service is based in the new Royal Cornhill Hospital, the main psychiatric teaching hospital in Aberdeen. The core staffing is a half-time psychiatrist, a half-time clinical psychologist, a dietician, three $G$ grade nurses and a secretary with variable hours from junior medical staff and an audit assistant. We defined our target patient group as those with anorexia nervosa, bulimia nervosa and related disorders where these were the main presenting problem. We advertised the service by sending leaflets to all general practitioners (GPs) in the area and to other potential referrars such as student counsellors and other mental health professionals.

\section{Clinical services}

Some patients are initially assessed by the psychologist or psychiatrist but most are allocated to one of the nurse therapists taking account of peripheral clinics which the nurses have been developing in the outlying parts of the Grampian area. We welcome direct enquiries from patients, relatives and friends, but strongly encourage referral through the GP and if the GP is not involved a psychiatrist sees the referral.

We wish to enable generalist colleagues to continue seeing patients and offer a consultative service to them but some managers seemed to believe that we should see all eating disorder patients. We had to explain that this is not logistically possible and not clinically necessary.

\section{Assessment}

We have tried to provide a "comprehensive multidimensional assessment" as recommended by the American Psychiatric Association (1993). This includes two to three sessions with the primary therapist, a nutritional assessment by the dietician, a physical assessment by a junior doctor and, if indicated, a psychiatric assessment by the consultant. Standard questionnaires are used to supplement information obtained at interview.

We use an innovative multi-disciplinary record and we had to spend some time overcoming the problem of harmonising practice. We did not realise how much the approach of the professions differed and how difficult it is to change established record-keeping practices. This remains a problem when junior psychiatrists pass through the service.

\section{Treatment}

The treatment is usually eclectic individual psychotherapy based on cognitive-behavioural principles but often with some exploration of relevant past adverse life-events. Relatives may be seen but the focus is on the individual, and for those able to come to Aberdeen there is parallel nutritional therapy with the dietician.

Three 12-session closed groups have been run successfully for bulimic disorders but there have been insufficient numbers of patients with anorexia nervosa to sustain a day programme. which we planned to run along the lines of the pioneering work at the Cullen Centre in Edinburgh (Freeman, 1992). 
The most common drug treatments used are antidepressants for bulimia or depression and nutritional supplements.

\section{Supervision and training}

Our nurses have had close supervision of clinical work from the consultant clinical psychologist, they have attended the one-year Dundee course in cognitive-behavioural therapy, and fully participate in a continuing professional development programme. The consultant psychiatrist provides supervision to the dietician and psychologist who also have a peer group structure within their own professions. In spite of the very high level of training, experience, supervision and competence of our nurse therapists a few referrers seem unhappy with a nurse assessment but most of the informal feedback is very positive.

\section{Teaching and consultancy}

All members of the team take a full part in providing education, training, supervision and liaison to mental health professionals, a local support/self-help group and other medical specialities.

\section{Research and audit}

There were pre-existing research interests, notably in epidemiology using the North East of Scotland Psychiatric Case Register (Crisp et al 1992; Eagles et ah, 1995). In addition we have begun to study the development of attitudes to weight and shape in childhood and adolescence, raised serum amylase in bulimia nervosa, early identification of eating disorders in general practice and binge eating disorder at an obesity clinic.

We have established a clinically detailed database on all our patients, which increases the possibilities for research and audit, and a summary of clinical activity to date is presented below.

\section{Clinical activity from January 1994 to December 1996}

There were 634 referrals, with $12.6 \%$ being uneventuated. The ICD-10 (World Health OrganIzation, 1992) diagnostic breakdown for 531 patients with a documented diagnosis at the end of 1996 is shown in Fig. 1, around half of all patients have bulimia nervosa or its partial syndrome (atypical bulimia nervosa). The first referral rate per 100000 total population of Grampian was 3.3 for anorexda nervosa (7.4 when atypical cases were included) and 10.9 for bulimia nervosa (14.2 when including atypical cases).

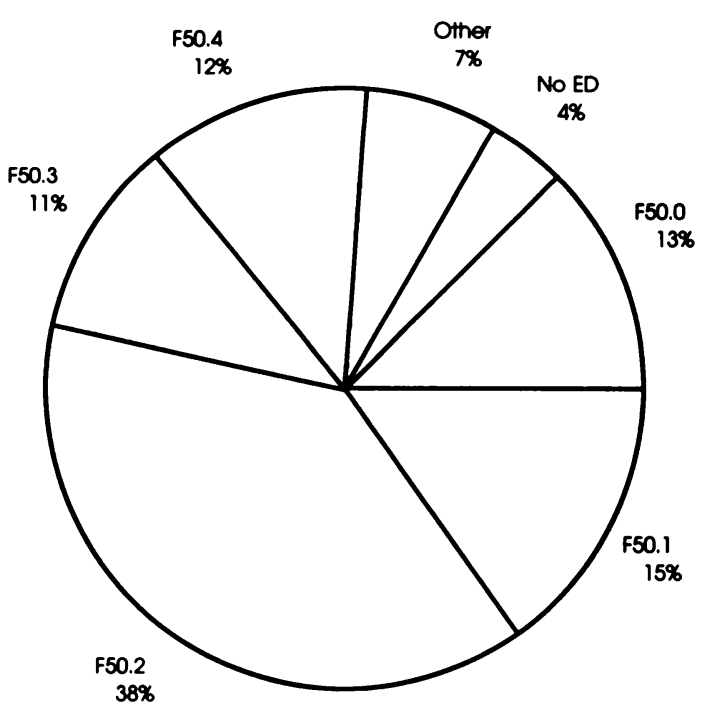

Fig. 1. ICD-10 diagnosis for patients at the end of 1996 (F50.0, anorexia nervosa; F50.1, atypical anorexia nervosa; F50.2, bulimia nervosa; F50.3, atypical bulimia nervosa; F50.4, overeating with psychological disturbance; Other, other eating disorders; No ED, no eating disorder).

Seventy per cent of referrals came from GPs, $19 \%$ from psychiatrists, just $2 \%$ were selfreferrals and the remainder came from a variety of other professional sources. As expected the majority of referrals are young women, with $85 \%$ aged under 35 and only $3.4 \%$ of referrals were male.

Ninety-five per cent of patients came from the Grampian Area, the remainder coming from the surrounding health board areas in the North of Scotland. The Grampian Health Board area was divided into three sectors for the provision of psychiatric services, namely the city, counties (the rural hinterland of Aberdeen) and Moray (around Elgin) catchment areas. Thirty-seven per cent of the population lives in the city catchment, but $67 \%$ of the Grampian referrals to the Eating Disorder Service come from the city.

Questionnaire data for the Eating Disorders Inventory showed a profile very similar to the eating disorder reference groups in the manual (EDI; Garner, 1991) and $77 \%$ had moderate or extreme levels of body shape concern on the Body Shape Questionnaire (BSQ; Cooper et al, 1987). Scores on the Hospital Anxiety and Depression Questionnaire (HAD; Zigmond \& 
Snaith, 1983) showed $69 \%$ had moderate or severe levels of anxiety and $36 \%$ had moderate or severe levels of depression.

\section{Comment}

Our findings show that there is a demand for specialist eating disorder services from patients with significant eating disorder psychopathology, anxiety and depression (i.e. there is not only a demand but also a need for their service). It is important for purchasers to note that the demands on the service are not only clinical, but also include frequent requests for teaching, consultation and advice. We also confirmed that it is essential to budget for staff training which is likely to be expensive, may require secondment to other centres and will be a recurring cost because of the need to sustain the service when staff move on. All staff should spend some time not working with eating disorders in order to reduce the risk of burn-out, but rigid administrative structures make this difficult to arrange once dedicated funding and staffing is established.

The mean annual referral rates of 3.3 per 100000 for tightly defined anorexia nervosa and 7.4 per 100000 when including atypical cases were in the same range of around five per 100000 total population in studies looking at referrals to mental health services elsewhere (Hoek, 1995). Our referral rate is also close to a recent incidence estimate of 4.2 per 100000 for anorexia nervosa found in a UK primary care study (Turnbull et al, 1996), suggesting that, in Grampian, the majority of cases of anorexia nervosa are referred for specialist help (bearing in mind that our study excluded patients under 18).

For bulimia nervosa our annual referral rate of 10.9 per 100000 was close to the primary care incidence found in Holland where a little over half are referred to mental health services (Hoek, 1995). This suggests that in north-east Scotland there is a higher prevalence, a lower threshold for referral, there are differences in diagnostic practice or a combination of these.

We found a large difference in referral rates between the city and rural areas which could be due to a higher concentration of young students in the city or a higher referral threshold for rural GPs, but there is also evidence from primary care studies in Holland that eating disorders, particularly bulimia nervosa, are more prevalent in urban areas (Hoek, 1991). We actively discouraged referrals bypassing GPs but our very low self-referral rate suggests that this policy may have blocked access for some patients.
Most of our therapeutic work is with normal weight bulimic patients but we have provided help for some overweight patients, particularly those with binge eating. We do not directly address weight reduction but we can see merit in more collaboration with colleagues who work with obesity.

Patients with severe, chronic anorexia nervosa present a therapeutic challenge which seems, at times, to frustrate and defeat our intensive outpatient treatment efforts. We have been unable to establish a dedicated day programme for these patients (which may reflect our geographically dispersed and relatively small population) and they usually do not do well in our general psychiatric wards. Reports from specialist units are more encouraging (Crisp et al, 1985) and we think there is a strong case for one or two carefully evaluated specialist in-patient units for anorexia nervosa in Scotland.

In conclusion, a specialist out-patient-based service such as ours goes a long way to meeting the clinical demand for adults with eating disorders and our initial experience should be useful to others planning local services. Looking to the future we need to consolidate what we have and, if resources permit, we would like to ensure a geographically more equitable provision of service, develop techniques for earlier identification and treatment, and investigate whether we can make a greater contribution to the increasing problem of obesity.

\section{Acknowledgements}

I thank patients and colleagues for the provision and collection of data, Lesley Michie for data analysis, Grampian Health Board and Grampian Healthcare NHS Trust for funding and Dr John Eagles for helpful comments on an early draft.

\section{References}

American Psychlatric Association (1993) Practice guideline for eating disorders. American Journal of Psychiatry. 150, 207-228.

COOPER, P. J., TAYLOR, M. J., COOPER, Z., et al (1987) The development and validation of the body shape questionnaire. International Journal of Eating Disorders, 6, 485-494.

Crisp, A. H., NorTon, K. R. S., JURCZAK, S., et al (1985) A treatment approach to anorexia nervosa-25 years on. Journal of Psychiatric Research, 19, 393-404.

-. Callender. J. S., HaleK, C., et al (1992) Long term mortality in anorexia nervosa. British Journal of Psychiatry, 161, 104-107.

EAgles, J. M., JohNSTON, M. I., HUNTER, D., et al (1995) Increasing incidence of anorexia nervosa in the female population of Northeast Scotland. American Journal of Psychiatry. 182, 1266-1271.

FREEMAN, C. (1992) Day patient treatment for anorexia nervosa. British Review of Bulimia and Anorexia Neruosa, 6, 2-9. 
GARNER, D. M. (1991) Eating Disorders Inventory-2. Professional Manual. Odessa, FL: Psychological Assessment Resources.

HOEK, H. W. (1991) The incidence and prevalence of anorexia nervosa and bulimia nervosa in primary care. Psychological Medicine, 21, 455-460.

- (1995) The distribution of eating disorders. In Eating Disorders and Obesity (eds K. D. Brownell \& C. G. Fairburn), pp. 207-211. New York: Guilford.

ROYAL COLLEGE OF PSYCHIATRISTS (1992) Eating Disorders (Council Report CR14). London: Royal College of Psychiatrists.

TURnbull, S., WARD, A., TrEasure, J., et al (1996) The demand for eating disorder care. An epidemiological study using the general practice research database. British Journal of Psychiatry, 169, 705-712.
WORLD HEALTH ORGANIZATION (1992) The ICD-10 Classification of Mental and Behavioural Disorders: Clinical Descriptions and Diagnostic Guidelines. Geneva: WHO.

Zigmond, A. S. \& SNATth, R. P. (1983) The hospital anxiety and depression scale. Acta Psychiatrica Scandinavica. 67, 361-370.

Harry R. Millar, Consultant Psychiatrist, Eating Disorder Service, Fulton Clinic, Royal Cornhill Hospital, Aberdeen AB25 2ZH

\title{
Twenty-eight years of liaison psychiatry at a general hospital
}

\author{
A. J. Carson, H. Dawson, D. Marshall and K. Slatford
}

\begin{abstract}
Aims and method A questionnaire was used to measure physician satisfaction with the unit and comparison of referral numbers and diagnoses with 1967. when the unit was new, and in 1985 when it was re-audited.

Results Despite physicians' satisfaction with the service, the referral rate remains unchanged.

Clinical Implications Luaison psychiatry should not concern itself with trying to increase referral rates. Research into patient outcome should be a priority and guide its future direction.
\end{abstract}

The liaison psychiatry unit to be described in this study was one of the first in Scotland. It was audited in its early days by Mcleod \& Walton (1969), and again 18 years later by Brown \& Waterhouse (1987). Despite the enthusiasm of the initial review, Brown \& Waterhouse showed that in some respects the unit had failed to live up to its earlier promise. In particular they were concerned that despite the fact that a large proportion of patients in a general hospital setting have been shown to have psychological morbidity (Shepherd et al, 1960; Maguire et al, 1974), only a small proportion were referred for psychiatric assessment. They, like many others, considered that the fault lay with psychiatrists style of relating to their colleagues. They conclude quoting Mason (1975) "that psychiatrists are seen as not readily available, remote in thought and tending to express opinions in a style which alienates physicians'". Ten years on we describe a further audit of liaison psychiatry at the Western General Hospital, Edinburgh.

The aims of the present study are to assess by questionnaire the views of the hospital's consultant staff on the quality of service provided by the department; to review referral numbers and diagnoses; and to compare these to the rates described in 1967 and 1985.

\section{The study}

Liaison psychiatry in the Western General Hospital

The study took place in the Department of Psychological Medicine of the Western General Hospital in Edinburgh. The Western General is a 600-bed teaching hospital in which most medical specialities are represented. It has been subject to much internal change over the past eight years as part of ongoing rationalisation of acute services within Lothian.

The Accident and Emergency Department, which took a third of emergencies from the city, became unsustainable after the closure of orthopaedic, paediatric, gynaecological and obstetric services and it closed in 1991. A Geriatric Assessment Unit was opened in 1990. 